\title{
Biological control using invertebrates and microorganisms: plenty of new opportunities
}

\author{
Joop C. van Lenteren · Karel Bolckmans · Jürgen Köhl • \\ Willem J. Ravensberg • Alberto Urbaneja
}

Received: 9 December 2016/ Accepted: 13 March 2017/Published online: 18 March 2017

(C) The Author(s) 2017. This article is an open access publication

\begin{abstract}
In augmentative biological control (ABC), invertebrate and microbial organisms are seasonally released in large numbers to reduce pests. Today it is applied on more than 30 million ha worldwide. Europe is the largest commercial market for invertebrate biological control agents, while North America has the largest sales of microbials. A strong growth in use of $\mathrm{ABC}$, particularly of microbial agents, is taking place in Latin America, followed by Asia. The current popularity of $\mathrm{ABC}$ is due to (1) its inherent positive
\end{abstract}

\section{Handling Editor: Russell Messing.}

Electronic supplementary material The online version of this article (doi:10.1007/s10526-017-9801-4) contains supplementary material, which is available to authorized users.

J. C. van Lenteren $(\varangle)$

Laboratory of Entomology, Wageningen University, P.O. Box 16, 6700 AA Wageningen, The Netherlands e-mail: Joop.vanLenteren@wur.nl

J. C. van Lenteren

Laboratory of Chemical Ecology and Insect Behavior, Department of Entomology and Acarology, ESALQ/USP, Piracicaba, SP, Brazil

K. Bolckmans

Biobest NV, Ilse Velden 18, 2260 Westerlo, Belgium

e-mail: Karel.Bolckmans@biobestgroup.com

J. Köhl

Wageningen University \& Research, Droevendaalsesteeg 1, 6708 PB, Wageningen, The Netherlands

e-mail: jurgen.kohl@wur.nl characteristics (healthier for farm workers and persons living in farming communities, no harvesting interval or waiting period after release of agents, sustainable as there is no development of resistance against arthropod natural enemies, no phytotoxic damage to plants, better yields and a healthier product, reduced pesticide residues [well below the legal Maximum Residue Levels (MRLs)], (2) professionalism of the biological control industry (inexpensive large scale mass production, proper quality control, efficient packaging, distribution and release methods, and availability of many (>440 species) control agents for numerous pests), (3) a number of recent successes showing how biological control can save agricultural production when pesticides fail or are not available, (4) several

\section{W. J. Ravensberg}

Koppert Biological Systems, Veilingweg 14, PO Box 155, 2650 AD Berkel en Rodenrijs, The Netherlands e-mail: wravensberg@koppert.nl

\section{A. Urbaneja}

Centro de Protección Vegetal y Biotecnología, Unidad de Entomología UJI-IVIA, Instituto Valenciano de Investigaciones Agrarias (IVIA), Carretera de MoncadaNáquera Km. 4,5, 46113 Moncada, Valencia, Spain e-mail: aurbaneja@ivia.es 
non-governmental organizations (NGOs), consumers, and retailers demanding pesticide residues far below the legal MRLs, and (5) policy developments in several regions of the world aimed at reduction and replacement of synthetic pesticides by more sustainable methods of pest management. We are convinced, however, that $\mathrm{ABC}$ can be applied on a much larger area than it is today. We plead in the short term for a pragmatic form of agriculture that is adaptable, nondogmatic and combines the sustainability gain from all types of agriculture and pest management methods. We then propose to move to "conscious agriculture", which involves participation of all stakeholders in the production and consumer chain, and respects the environment and resource availability for future generations. Were "conscious agriculture" to be considered a serious alternative to conventional farming, $\mathrm{ABC}$ would face an even brighter future.

Keywords Augmentative biological control - Pest control policies - Benefits of biological control . Market developments in biological control . Worldwide use of biological control - Integrated pest management $\cdot$ Conscious agriculture

\section{Introduction}

Politicians, policy makers, retailers, consumers, growers and grower organizations are increasingly asking for and speaking about biological control. Hardly a day passes during which we, the authors of this paper, do not receive a question on how to control a certain pest, disease or weed, where to obtain biological control agents, and how to stimulate use of this environmentally safe pest management method. The European Union (EU) has been advocating the use of biological control since 2009 in its Sustainable Use of Pesticides Directive (EC 2009). The President of China recently launched a "National research program on reduction in chemical pesticides and fertilizers in China" involving more than 340 million US\$, indicating a need for the development and application of non-chemical control methods. Together, the authors of this paper have been working in the field of augmentative biological control (ABC) for more than 150 years. We noted a hesitant start to $\mathrm{ABC}$ in the 1970 s, then a burst of activity took place over the next 25 years. During the first decade of the twenty-first century fewer new biological control agents came to the market, but during the second decade we again experienced a new phase with strong growth in both the development of new agents and a market for biological control (van Lenteren 2012; Tables 1, 2 and 3 in this paper).

Simply said, biological control is the use of a population of one organism to reduce the population of another organism. Biological control has been in use for at least 2000 years, but modern use started at the end of the nineteenth century (DeBach 1964; van Lenteren and Godfray 2005). Four different types of biological control are known: natural, conservation, classical, and augmentative biological control (Eilenberg et al. 2001; Cock et al. 2010). Natural biological control is an ecosystem service (Millennium Ecosystem Assessment 2005) whereby pest organisms are reduced by naturally occurring beneficial organisms. This occurs in all of the world's ecosystems without any human intervention, and, in economic terms, is the greatest contribution of biological control to agriculture (Waage and Greathead 1988). Conservation biological control consists of human actions that protect and stimulate the performance of naturally occurring natural enemies. This form of biological control is currently receiving a lot of attention for pest control. Conservation biological control of plant diseases is focused on the role of the natural microbiome in suppressing plant diseases in soil and crop residues, and of the natural microbiome in and on plants in providing resilience to pest and pathogen infection (Mendes et al. 2011; Weller et al. 2002). In classical biological control, natural enemies are collected in an exploration area (usually the area of origin of the pest) and then released in areas where the pest is invasive, often resulting in permanent pest population reduction and enormous economic benefits (see Cock et al. 2010). As this was the first type of biological control deliberately and widely practiced, it is called "classical" biological control (DeBach 1964). In augmentative biological control (ABC), natural enemies (parasitoids, predators or micro-organisms) are mass-reared for release in large numbers either to obtain immediate control of pests in crops with a short production cycle (inundative biological control) or for control of pests during several generations in crops with a long production cycle (seasonal inoculative biological control) (Cock et al. 2010; Lorito et al. 2010; Parnell et al. 2016; van Lenteren 2012). 
This paper is focussed on augmentative biological control (ABC). It has been applied with success for more than 100 years in several cropping systems (Gurr and Wratten 2000; Cock et al. 2010). In this paper, we illustrate (1) the important role $\mathrm{ABC}$ is playing today, (2) how many biological control agents are commercially available and against which pests they are applied, (3) in what way ABC can result in cleaner, greener, healthier and more sustainable agriculture through policy measures and regulations, and, finally, (4) the need for a type of agriculture that respects the environment and optimizes use of ecosystem services.

Note: in this paper we often use the word 'pest' as defined by FAO/IIPC (1997), which includes animal pests, weeds and diseases.

\section{Where is augmentative biological control currently applied?}

Large scale regular releases and mass production of natural enemies means that $\mathrm{ABC}$ is often a commercial activity (van Lenteren 2012). ABC is thought to have been used for the first time in China around $300 \mathrm{AD}$ (van Lenteren and Godfray 2005). Modern ABC started in the 1880s with the use of the insect pathogen Metarhizium anisopliae by Metchnikoff in Russia for control of beetles in various crops (MacBain Cameron 1973). Today, ABC is applied in many areas of agriculture, such as fruit and vegetable crops, cereals, maize, cotton, sugarcane, soybean, grapes and many greenhouse crops (Table 1), and is often part of an

Table 1 Worldwide use of major augmentative biological control programs (after van Lenteren and Bueno 2003), with updates and supported with references when large differences in areas under control existed between 2003 and 2016

\begin{tabular}{lll}
\hline Natural enemy & Pest and crop & Area under control (in ha) \\
\hline Trichogramma spp. & Lepidopteran pests in vegetables, cereals, cotton & 10 million, former USSR \\
Trichoderma spp. & Soil diseases various crops & 5 million, Brazil, Europe \\
Trichogramma spp. & Lepidopteran pests in various crops, forests & 4 million, China \\
Cotesia spp. & Sugarcane borers & 3.6 million, South America, China \\
Metarhizium anisoplae & Lepidopteran pests in sugar cane & 2 million, Brazil \\
Trichogramma spp. & Lepidopteran pests in corn, cotton, sugarcane, tobacco & 1.5 million, Mexico \\
Trichogramma spp. & Lepidopteran pests in cereals, cotton, sugarcane, pastures & 1.2 million, South America \\
AgMNPV & Soybean caterpillar in soybean & 1 million, Brazil \\
Beauvaria bassiana & Coffee berry borer in coffee, whitefly in several crops & 1 million, Brazil \\
Entomopathogenic fungi & Coffee berry borer in coffee & 0.55 million, Colombia \\
Trichogramma spp. & Lepidopteran pests in cereals and rice & 0.3 million, SouthEast Asia \\
Trichogramma spp. & Lepidopteran pests in sugar cane and tomato & 0.3 million, NorthEast Africa \\
Predatory mites & Spider mites in greenhouses, fruit orchards, tea and cotton & 0.07 million China \\
Trichogramma spp. & Ostrinia nubilalis in corn & 0.05 million, Europe \\
Orgilus sp. & Pine shooth moth, pine plantations & 0.05 million, Chile \\
$>30$ spp. of nat. enemies & Many pests in greenhouses and interior plant-scapes & 0.05 million, worldwide \\
Egg parasitoids & Soybean stinkbugs in soybean & 0.03 million, South America \\
Five spp. of nat. enemies & Lepidoptera, Hemiptera, spider mites in orchards & 0.03 million, Europe \\
\hline
\end{tabular}

\footnotetext{
${ }^{a}$ Recent data about use of Trichogramma in Russia were not available

b Bettiol W and Pedrazzoli D, personal communication 2016

c Liu et al. (2014)and Wang et al. (2014)

d Parra JRP and Pedrazzoli D, personal communication 2016

e Bettiol W and Pedrazzoli D, personal communication 2016

${ }^{\mathrm{f}}$ Bettiol W and Parra JRP, personal communication 2016

g Aristizabal et al. (2016)

h Yang et al. (2014)
} 
Table 2 Additional natural enemy species to the table of van Lenteren (2012), "Commercial availability of invertebrate natural enemies used worldwide in augmentative biological control, with region of use, year of first use and market value."

\begin{tabular}{|c|c|c|c|c|c|}
\hline Natural enemy & Classification & Region where used $^{b}$ & Target(s) & $\begin{array}{l}\text { Year of first } \\
\text { use }\end{array}$ & $\begin{array}{l}\text { Market } \\
\text { value }^{\mathrm{a}}\end{array}$ \\
\hline Adalia spp. & Coleoptera & Latin America & Aphids & 1941 & M \\
\hline Ageniaspis citricola & Hymenoptera & Latin America & Lepidopterans & 1998 & M \\
\hline Allotropa convexifrons & Hymenoptera & Europe & Pseudococcids & 2005 & $\mathrm{~S}$ \\
\hline Allotropa musae & Hymenoptera & Europe & Pseudococcids & 2006 & $\mathrm{~S}$ \\
\hline Amblydromalus limonicus & Acari & Europe & $\begin{array}{l}\text { Thrips, whiteflies, } \\
\text { tarsonomids }\end{array}$ & 2013 & $\mathrm{~L}$ \\
\hline Amblyseius aizawai & Acari & Asia & Mites & 1992 & M \\
\hline Amblyseius longispinosus & Acari & Asia & Mites & 1990 & $\mathrm{~S}$ \\
\hline Amblyseius makuwa & Acari & Asia & Mites & 1991 & $\mathrm{~S}$ \\
\hline Amblyseius mckenziei & Acari & Europe & Mites & 1985 & $\mathrm{~S}$ \\
\hline Amblyseius nicholsi & Acari & Asia & Mites in citrus & 1980 & $\mathrm{~L}$ \\
\hline Amblyseius spp. & Acari & Australia & Mites in citrus & 1990 & $\mathrm{~L}$ \\
\hline Anagyrus kamali & Hymenoptera & Latin America & Pseudococcids & 1990 & $\mathrm{~S}$ \\
\hline Anagyrus sinope & Hymenoptera & Europe & Pseudococcids & 2006 & $\mathrm{~S}$ \\
\hline Anaphes nitens & Hymenoptera & Europe & Coleopterans & 1995 & $\mathrm{~S}$ \\
\hline Anastatus japonicus & Hymenoptera & Asia & Hemipterans & 1970 & $\mathrm{~L}$ \\
\hline Anastatus sp. & Hymenoptera & Asia, Australia & Hemipterans & 2010 & $\mathrm{~S}$ \\
\hline Anastatus tenuipes & Hymenoptera & North America & Cockroaches & 1970 & S \\
\hline Androlaelaps casalis & Acari & Europe & Mites on vertebrates & 2008 & M \\
\hline Anisopteromalus calandrae & Hymenoptera & $\begin{array}{l}\text { Europe, North } \\
\text { America }\end{array}$ & Coleopterans & 1990 & $\mathrm{~S}$ \\
\hline Aphidius sp. & Hymenoptera & Latin America & Aphids & 1980 & $\mathrm{~S}$ \\
\hline Billaea claripalpis & Diptera & Latin America & Lepidopterans & 1976 & $\mathrm{~S}$ \\
\hline Bracon brevicornis & Hymenoptera & Europe & Lepidopterans & 2000 & S \\
\hline Cephalonomia tarsalis & Hymenoptera & Europe & Coleopterans & 1995 & $\mathrm{~S}$ \\
\hline Ceraeochrysa cincta & Neuroptera & Latin America & Aphids & 1990 & $\mathrm{~S}$ \\
\hline Ceraeochrysa smithi & Neuroptera & Latin America & Aphids & 1995 & $\mathrm{~S}$ \\
\hline Cheyletus eruditus & Acari & Europe & Vertebrate mites & 2004 & $\mathrm{~S}$ \\
\hline Chouioia cunea & Hymenoptera & Asia & Lepidopterans & 2005 & M \\
\hline Chrysoperla asoralis & Neuroptera & Latin America & Aphids & 1990 & $\mathrm{~S}$ \\
\hline Chrysoperla cinta & Neuroptera & Latin America & Aphids & 1990 & $\mathrm{~S}$ \\
\hline Chrysoperla comanche & Neuroptera & North America & Aphids & 1990 & M \\
\hline Chrysoperla lucasina & Neuroptera & Europe & Aphids & 1995 & M \\
\hline Chrysoperla $(=$ Chrysopa $)$ sinica & Neuroptera & Asia & Aphids, lepidopterans & 2000 & M \\
\hline Coccidophilus citricola & Coleoptera & $\begin{array}{l}\text { Latin America, } \\
\text { Europe }\end{array}$ & Diaspidids & 1982 & $\mathrm{~S}$ \\
\hline Coccidoxenoides peregrinus & Hymenoptera & $\begin{array}{l}\text { North and Latin } \\
\text { America }\end{array}$ & $\begin{array}{l}\text { Diaspidids, } \\
\text { pseudococcids }\end{array}$ & 2006 & $\mathrm{~S}$ \\
\hline Comperia merceti & Hymenoptera & North America & Cockroaches & 1980 & $\mathrm{~S}$ \\
\hline Copidosoma sp. & Hymenoptera & Latin America & Lepidopterans & 1995 & $\mathrm{~S}$ \\
\hline Cotesia marginiventris & Hymenoptera & North America & Lepidopterans & 1990 & $\mathrm{~S}$ \\
\hline Cotesia plutellae & Hymenoptera & North America & lepidopterans & 1995 & M \\
\hline Cryptolaemus montrouzieri & Coleoptera & $\begin{array}{l}\text { Europe, South } \\
\text { America }\end{array}$ & Mealybugs & 1927 & M \\
\hline
\end{tabular}


Table 2 continued

\begin{tabular}{|c|c|c|c|c|c|}
\hline Natural enemy & Classification & Region where used ${ }^{b}$ & Target(s) & $\begin{array}{l}\text { Year of first } \\
\text { use }\end{array}$ & $\begin{array}{l}\text { Market } \\
\text { value }^{\mathrm{a}}\end{array}$ \\
\hline Cycloneda limbifer & Colecoptera & Europe & Aphids & 1990 & $\mathrm{~S}$ \\
\hline Diachasmimorpha longicaudata & Hymenoptera & Latin America & Dipterans & 1990 & M \\
\hline Dibrachys cavus & Hymenoptera & Europe & Dipterans & 1990 & $\mathrm{~S}$ \\
\hline Dirhinus giffardii & Hymenoptera & Latin America & Dipterans & 1990 & $\mathrm{~S}$ \\
\hline Elasmus albipennis & Hymenoptera & Europe & Lepidopterans & 1995 & $\mathrm{~S}$ \\
\hline Encarsia perniciosi & Hymenoptera & Europe & Scales & 1932 & $\mathrm{~L}$ \\
\hline Encarsia sp. & Hymenoptera & Latin America & Whiteflies & 1995 & $\mathrm{~S}$ \\
\hline Ephedrus cerasicola & Hymenoptera & Europe & Aphids & 2008 & $\mathrm{~L}$ \\
\hline Ephedrus plagiator & Hymenoptera & Europe & Aphids & 2010 & M \\
\hline Eretmocerus hayati & Hymenoptera & Australia & Whiteflies & 2006 & M \\
\hline Eriopsis connexa & Coleoptera & Latin America & $\begin{array}{l}\text { Coccids, Aphids, } \\
\text { hemipterans }\end{array}$ & 2000 & $\mathrm{~S}$ \\
\hline Eucanthecona furcellata & Hemiptera & Asia & Aphids, lepidopterans & 1996 & $\mathrm{~S}$ \\
\hline Euseius gallicus & Acari & Europe & Thrips, whitefly & 2013 & M \\
\hline Euseius ovalis & Acari & Europe & Thrips, whitefly & 2008 & M \\
\hline Euseius stipulatus & Acari & $\begin{array}{l}\text { Europe, South } \\
\text { America }\end{array}$ & Mites & 2006 & M \\
\hline Forficula sp. & Dermaptera & Asia & Lepidopterans & 2010 & $\mathrm{~S}$ \\
\hline $\begin{array}{l}\text { Galendromus (Metaseiulus) } \\
\text { annectens }\end{array}$ & Acari & North America & Mites & 1990 & M \\
\hline $\begin{array}{l}\text { Galendromus (Metaseiulus) } \\
\text { helveolus }\end{array}$ & Acari & North America & Mites & 1999 & $\mathrm{~S}$ \\
\hline Galendromus (Metaseiulus) pyri & Acari & North America & Mites & 1995 & $\mathrm{~L}$ \\
\hline Galeolaelaps gillespieii & Acari & North America & Dipterans, thrips & 2010 & $\mathrm{~L}$ \\
\hline Geocoris punctipes & Hemiptera & $\begin{array}{l}\text { North and Latin } \\
\text { America }\end{array}$ & Lepidopterans, whiteflies & 2000 & $\mathrm{~S}$ \\
\hline Gynaeseius liturivorus & Acari & Asia & Thrips, whitefly & 2013 & M \\
\hline Habrobracon sp. & Hymenoptera & Latin America & Lepidopterans & 1986 & $\mathrm{~S}$ \\
\hline Haplothrips brevitubus & Thysanoptera & Asia & Thrips & 2010 & $S$ \\
\hline Heterorhabditis indica & Nematoda & North America & Coleopterans, dipterans & 2000 & $\mathrm{~S}$ \\
\hline Hydrotaea aenescens & Diptera & $\begin{array}{l}\text { Europe, North } \\
\text { America }\end{array}$ & Dipterans & 2000 & $\mathrm{~S}$ \\
\hline Lariophagus distinguendus & Hymenoptera & Europa & Coleopterans & 1995 & $S$ \\
\hline Leis (Harmonia) dimidiata & Coleoptera & Europe & Aphids & 1995 & $\mathrm{~S}$ \\
\hline Leminia biplagiata & Coleoptera & Asia & Aphids, whiteflies & 1998 & $S$ \\
\hline Leptomastix algirica & Hymenoptera & Europe & Pseudococcids & 2011 & $\mathrm{~S}$ \\
\hline Leptopilina heterotoma & Hymenoptera & Europe & Dipterans & 2007 & $\mathrm{~S}$ \\
\hline Lydella jalisco & Diptera & Latin America & Lepidopterans & 1996 & $\mathrm{~S}$ \\
\hline Macrocentrus prolificus & Hymenoptera & Latin America & Lepidopterans & 2005 & $\mathrm{~S}$ \\
\hline Mallada basalis & Neuroptera & Asia & Aphids, thrips, etc. & 2000 & M \\
\hline Mantis religiosa & Mantodea & North America & Many pests & 1970 & $\mathrm{~S}$ \\
\hline Megastigmus brevivalvus & Hymenoptera & Australia & Hymenopterans & 1995 & $\mathrm{~S}$ \\
\hline Megastigmus trisulcus & Hymenoptera & Australia & Hymenopterans & 1995 & $\mathrm{~S}$ \\
\hline Menochilus sexmaculatus & Coleoptera & Asia & Aphids, whiteflies & 2010 & $\mathrm{~S}$ \\
\hline Metagonistylum minense & Diptera & Latin America & Lepidopterans & 1980 & $\mathrm{~S}$ \\
\hline
\end{tabular}


Table 2 continued

\begin{tabular}{|c|c|c|c|c|c|}
\hline Natural enemy & Classification & Region where used ${ }^{\mathrm{b}}$ & Target(s) & $\begin{array}{l}\text { Year of first } \\
\text { use }\end{array}$ & $\begin{array}{l}\text { Market } \\
\text { value }^{\mathrm{a}}\end{array}$ \\
\hline Micromus variegatus & Neuroptera & North America & Aphids & 2010 & $\mathrm{~L}$ \\
\hline Necremnus artynes & Hymenoptera & Europe & Lepidopterans & 2010 & $\mathrm{~S}$ \\
\hline Neodryinus typhlocybae & Hymenoptera & Europe & Planthoppers & 2007 & $\mathrm{~S}$ \\
\hline Neoseiulus (Amblyseius) barkeri & Acari & $\begin{array}{l}\text { Europe, Latin } \\
\text { America }\end{array}$ & Thrips & 1981 & $\mathrm{~S}$ \\
\hline Neoseiulus longispinosus & Acari & Latin America & Mites & 2005 & $\mathrm{~S}$ \\
\hline Nephus quadrimaculatus & Coleoptera & Europe & Aphids, pseudococcids & 2005 & $\mathrm{~S}$ \\
\hline Olla abdominalis (=v-nigrans) & Coleoptera & $\begin{array}{l}\text { North and Latin } \\
\text { America }\end{array}$ & Aphids, hemipterans & 1990 & $\mathrm{~S}$ \\
\hline Orius sauteri & Hemiptera & Asia & Aphids, mites, thrips, & 2005 & M \\
\hline Orius vicinus & Hemiptera & New Zealand & Thrips, aphids, mites & 2010 & M \\
\hline Pentalitomastix plethoricus & Hymenoptera & North America & Lepidopterans & 1980 & $\mathrm{~S}$ \\
\hline Peristenus relictus & Hymenoptera & North America & Hemipterans & 2001 & $\mathrm{~L}$ \\
\hline Podisus sp. & Hemiptera & Latin America & Lepidopterans & 1985 & $S$ \\
\hline Praon sp. & Hymenoptera & Latin America & Aphids & 1980 & $\mathrm{~S}$ \\
\hline Propylaea japonica & Coleoptera & Asia & Aphids & 2014 & $\mathrm{~S}$ \\
\hline $\begin{array}{l}\text { Propylaea } \\
\quad \text { quatuordecimpunctata }\end{array}$ & Coleoptera & Europe & Aphids & 1995 & $\mathrm{~S}$ \\
\hline Scymnus loewii & Coleoptera & New Zealand & Aphids & 1995 & $\mathrm{~S}$ \\
\hline Sphaerophoria rueppellii & Diptera & Europe & Aphids & 2015 & $S$ \\
\hline Stagmomantis carolina & Mantodea & North America & Many pest species & 1990 & $S$ \\
\hline Steinernema scapterisci & Nematoda & North America & Orthopterans & 1990 & $\mathrm{~S}$ \\
\hline Stethorus punctipes & Coleoptera & North America & Mites & 1980 & $\mathrm{~S}$ \\
\hline Stethorus sp. & Coleoptera & Latin America & Mites & 1995 & $\mathrm{~S}$ \\
\hline Sympherobius barberi & Neuroptera & North America & $\begin{array}{l}\text { Pseudococcids, aphids, } \\
\text { etc. }\end{array}$ & 1980 & $\mathrm{~L}$ \\
\hline Sympherobius maculipennis & Neuroptera & Latin America & Pseudococcids & 1990 & $\mathrm{~S}$ \\
\hline Sympherobius sp. & Neuroptera & Latin America & Whiteflies & 1995 & $\mathrm{~S}$ \\
\hline Synopeas sp. & Hymenoptera & Latin America & Dipterans & 1990 & $\mathrm{~S}$ \\
\hline Tamarixia radiata & Hymenoptera & Latin America & Psyllids & 2010 & $\mathrm{~L}$ \\
\hline Tamarixia triaozae & Hymenoptera & $\begin{array}{l}\text { North and Latin } \\
\text { America }\end{array}$ & Psyllids & 2001 & $\mathrm{~L}$ \\
\hline Telenomus podisi & Hymenoptera & Latin America & Hemipterans & 2004 & M \\
\hline Telenomus sp. & Hymenoptera & Latin America & Lepidopterans & 1990 & $\mathrm{~S}$ \\
\hline Tenodera aridifolia sinensis & Mantodea & North America & Many pest species & 1990 & S \\
\hline Tetrastichus hagenowi & Hymenoptera & Asia & Cockroaches & 1980 & S \\
\hline Tetrastichus howardii & Hymenoptera & Latin America & Lepidopterans & 1995 & $\mathrm{~S}$ \\
\hline Thyphiodromus pyri & Acari & Latin America & Mites & 2000 & M \\
\hline $\begin{array}{l}\text { Transeius }(=\text { Amblyseius }) \\
\text { montdorensis }\end{array}$ & Acari & Europe & $\begin{array}{l}\text { Thrips, whiteflies, } \\
\text { tarsonomids }\end{array}$ & 2004 & $\mathrm{~S}$ \\
\hline Trichogramma achaeae & Hymenoptera & Europe & Lepidopterans & 2012 & M \\
\hline Trichogramma bactrae & Hymenoptera & Latin America, Asia & Lepidopterans & 1980 & M \\
\hline $\begin{array}{l}\text { Trichogramma confusum } \\
\quad(=\text { chilonus })\end{array}$ & Hymenoptera & Asia, Australia & Lepidopterans & 1970 & $\mathrm{~L}$ \\
\hline Trichogramma embryophagum & Hymenoptera & Europe & Lepidopterans & 1994 & M \\
\hline Trichogramma euproctidis & Hymenoptera & Europe & Lepidopterans & 1960 & M \\
\hline
\end{tabular}


Table 2 continued

\begin{tabular}{|c|c|c|c|c|c|}
\hline Natural enemy & Classification & Region where used $^{\mathrm{b}}$ & Target(s) & $\begin{array}{l}\text { Year of first } \\
\text { use }\end{array}$ & $\begin{array}{l}\text { Market } \\
\text { value }^{\mathrm{a}}\end{array}$ \\
\hline Trichogramma fuentesi & Hymenoptera & Latin America & Lepidopterans & 1990 & $\mathrm{~S}$ \\
\hline Trichogramma japonicum & Hymenoptera & Asia & Lepidopterans & 1990 & $\mathrm{~S}$ \\
\hline Trissolcus basalis & Hymenoptera & Latin America & Hemipterans & 1995 & $\mathrm{~S}$ \\
\hline Typhlodromus occidentalis & Acari & Australia & Mites & 1970 & M \\
\hline Wollastoniella rotunda & Hemiptera & Asia & Thrips & 2005 & $\mathrm{~S}$ \\
\hline Xenostigmus bifasciatus & Hymenoptera & Latin America & Aphids & 2002 & $\mathrm{~S}$ \\
\hline Xylocoris flavipes & Hemiptera & North America & Coleopterans & 2000 & $\mathrm{~S}$ \\
\hline
\end{tabular}

A table listing all species used in biological control of invertebrates is provided as Supplementary electronic information

a Market value: $L$ large (hundred thousand to millions of individuals sold per week), $M$ medium (ten thousand to a hundred thousand individuals sold per week), $S$ small (hundreds to a few thousands individuals sold per week) In case of doubt, when numbers sold per week could not be estimated from published material, the market value was rated as $S$

${ }^{\mathrm{b}}$ Africa North $=$ North of Sahara; Africa South $=$ South of Sahara; North America = Canada + USA; Latin America = the Caribbean, Central and South America

Table 3 Registered microbial biological control agents for augmentative biological control in Australia (AUS), Brazil (BR), Canada (CA), European Union (EU), Japan (J), New Zealand (NZ) and United States of America (USA)

\begin{tabular}{|c|c|c|c|}
\hline Microorganism $^{\mathrm{a}}$ & $\begin{array}{l}\text { Type }^{\mathrm{b}} \text { of } \\
\text { organism }\end{array}$ & $\begin{array}{l}\text { Country/region where } \\
\text { approved }\end{array}$ & Target(s) \\
\hline Adoxophyes orana GV V-0001 & V & EU, J & Summer fruit tortrix \\
\hline Agrobacterium radiobacter & $\mathrm{B}$ & NZ (1975) & Crown gall \\
\hline Agrobacterium radiobacter $\mathrm{K} 1026$ & B & USA & Crown gall \\
\hline Agrobacterium radiobacter $\mathrm{K} 84$ & $\mathrm{~B}$ & CA, J, USA & Crown gall \\
\hline Alternaria destruens 059 & $\mathrm{~F}$ & USA & Cuscuta spp. (dodder) \\
\hline Ampelomyces quisqualis AQ10 & $\mathrm{F}$ & EU, USA & Powdery mildew \\
\hline Anagrapha falcifera NPV & $\mathrm{V}$ & USA & Anagrapha falcifera \\
\hline Anticarsia gemmatalis NPV & V & BR & Anticarsia gemmatalis \\
\hline Aspergillus flavus NRRL 21882 & $\mathrm{~F}$ & BR, USA & Aspergillus flavus mycotoxine \\
\hline Aspergillus flavus AF36 & $\mathrm{F}$ & USA & Aspergillus flavus mycotoxine \\
\hline $\begin{array}{l}\text { Aureobasidium pullulans DSM } 14940 \text { and DSM } \\
14941\end{array}$ & $\mathrm{Y}$ & EU, CA & $\begin{array}{l}\text { Bacterial and fungal flower and foliar } \\
\text { diseases }\end{array}$ \\
\hline Autographa californica NPV & V & CA & Autographa californica \\
\hline $\begin{array}{l}\text { Bacillus amyloliquefaciens (formerly B. subtilis) MBI } \\
600\end{array}$ & B & $\begin{array}{l}\text { CA, J, EU }{ }^{\mathrm{c}}, \mathrm{NZ} \\
(2009,2012), \mathrm{USA}\end{array}$ & Seed treatment, soil borne diseases \\
\hline Bacillus amyloliquefaciens $\mathrm{AH} 2$ & $\mathrm{~B}$ & $\mathrm{EU}^{\mathrm{c}}$ & Fungal soil diseases \\
\hline Bacillus amyloliquefaciens AT-332 & $\mathrm{B}$ & $\mathrm{J}$ & Botrytis, powdery mildew \\
\hline Bacillus amyloliquefaciens bs $1 \mathrm{~b}$ & $\mathrm{~B}$ & NZ (2010) & Foliar diseases \\
\hline Bacillus amyloliquefaciens PTA-4838 & B & USA & Nematodes \\
\hline $\begin{array}{l}\text { Bacillus amyloliquefaciens ssp. plantarum (syn. } \\
\text { Bacillus subtilis var. amyloliquefaciens) D747 }\end{array}$ & B & CA, EU, J, NZ (2010) & Seedling fungal pathogens \\
\hline Bacillus cereus BP01 & $\mathrm{B}$ & USA & Foliar plant growth regulator \\
\hline Bacillus firmus i-1582 & $\mathrm{B}$ & CA, EU, NZ (2016) & Nematodes \\
\hline Bacillus licheniformis SB3086 & $\mathrm{B}$ & USA & Fungal foliar diseases \\
\hline Bacillus mycoides J CX-10244 & B & CA, USA & Cercospora leaf spot on sugar beet \\
\hline
\end{tabular}


Table 3 continued

Microorganisma Type $^{\mathrm{b}}$ of Country/region where Target(s) organism approved

\begin{tabular}{|c|c|c|c|}
\hline Bacillus popilliae & $\mathrm{B}$ & USA & Japanese beetle \\
\hline Bacillus pumilus GB34 & $\mathrm{B}$ & USA & Root diseases of soy beans \\
\hline Bacillus pumilus QST 2808 & $\mathrm{~B}$ & BR, EU, USA & Fungal foliar diseases \\
\hline Bacillus subtilis ATCC 6051 & $\mathrm{~B}$ & NZ (2012) & Fungal foliar diseases \\
\hline Bacillus subtilis GB03 & $\mathrm{B}$ & CA, USA & Fungal diseases \\
\hline Bacillus subtilis HAI-0404 & $\mathrm{B}$ & $\mathrm{J}$ & Foliar diseases \\
\hline Bacillus subtilis IAB/BS03 & B & $\mathrm{EU}^{\mathrm{c}}$ & Foliar fungal and bacterial diseases \\
\hline Bacillus subtilis $\mathrm{KTSB}$ & $\mathrm{B}$ & NZ (2008) & Foliar diseases \\
\hline Bacillus subtilis QST 713 & $\mathrm{~B}$ & $\begin{array}{l}\text { BR, CA, EU, J, NZ } \\
\text { (2001), USA }\end{array}$ & Fungal foliar diseases \\
\hline Bacillus subtilis var. amyloliquefaciens FZB24 & B & $\mathrm{CA}, \mathrm{EU}^{\mathrm{c}}, \mathrm{USA}$ & Fungal foliar diseases \\
\hline Bacillus subtilis Y 1336 & $\mathrm{~B}$ & $\mathrm{~J}$ & Botrytis, powdery mildew \\
\hline Bacillus thuringiensis EG-7826 & $\mathrm{B}$ & $\mathrm{BR}$ & Lepidopteran caterpillars \\
\hline Bacillus thuringiensis BMP 123 & $\mathrm{~B}$ & $\mathrm{BR}$ & Lepidopteran caterpillars \\
\hline $\begin{array}{l}\text { Bacillus thuringiensis CryC encapsulated in killed } \\
\text { Pseudomonas fluorescens }\end{array}$ & B & USA & Lepidopteran caterpillars \\
\hline $\begin{array}{l}\text { Bacillus thuringiensis CrylA(c) and CrylC in killed } \\
\text { Pseudomonas fluorescens }\end{array}$ & $\mathrm{B}$ & USA & Lepidopteran caterpillars \\
\hline Bacillus thuringiensis EG 2348 & $\mathrm{~B}$ & $\mathrm{BR}, \mathrm{EU}$ & Lepidopteran caterpillars \\
\hline Bacillus thuringiensis SA-11 & $\mathrm{B}$ & $\mathrm{BR}, \mathrm{CA}, \mathrm{EU}$ & Lepidopteran caterpillars \\
\hline Bacillus thuringiensis SA-12 & B & $\mathrm{BR}, \mathrm{CA}, \mathrm{EU}$ & Lepidopteran caterpillars \\
\hline Bacillus thuringiensis Serotype H-14 & $\mathrm{B}$ & $\mathrm{CA}$ & Lepidopteran caterpillars \\
\hline Bacillus thuringiensis ssp. aizawai & $\mathrm{B}$ & $\mathrm{CA}$ & Lepidopteran caterpillars \\
\hline Bacillus thuringiensis ssp. aizawai & $\mathrm{B}$ & AUS (2000) & Lepidopteran caterpillars \\
\hline Bacillus thuringiensis ssp. aizawai ABTS-1857 & B & EU, NZ (1999) & Lepidopteran caterpillars \\
\hline Bacillus thuringiensis ssp. aizawai NB200 & $\mathrm{B}$ & USA & Lepidopteran caterpillars \\
\hline Bacillus thuringiensis ssp. aizawai GC-91 & $\mathrm{B}$ & USA & Lepidopteran caterpillars \\
\hline Bacillus thuringiensis ssp. aizawai GC-91 & B & $\mathrm{BR}, \mathrm{EU}$ & Lepidopteran caterpillars \\
\hline Bacillus thuringiensis ssp. aizawai/kurstaki & $\mathrm{B}$ & NZ (1995) & Lepidopteran caterpillars \\
\hline Bacillus thuringiensis ssp. galleriae SDS-502 & $\mathrm{B}$ & $\mathrm{CA}$ & Beetles \\
\hline Bacillus thuringiensis ssp. israelensis & B & USA & Mosquitoes \\
\hline Bacillus thuringiensis ssp. israelensis EG2215 & B & USA & Mosquitoes \\
\hline $\begin{array}{l}\text { Bacillus thuringiensis ssp. israeliensis (serotype } \\
\text { H-14) AM65-52 }\end{array}$ & $\mathrm{B}$ & $\mathrm{CA}, \mathrm{EU}$ & Mosquitoes \\
\hline Bacillus thuringiensis ssp. kurstaki & B & $\begin{array}{l}\text { AUS (1994), BR, EU, } \\
\text { J, NZ, USA }\end{array}$ & Lepidopteran caterpillars \\
\hline Bacillus thuringiensis ssp. kurstaki ABTS 351 & B & $\mathrm{EU}$ & Lepidopteran caterpillars \\
\hline Bacillus thuringiensis ssp. kurstaki PB 54 & $\mathrm{~B}$ & $\mathrm{EU}$ & Lepidopteran caterpillars \\
\hline Bacillus thuringiensis ssp. kurstaki (ALL STRAINS) & $\mathrm{B}$ & $\mathrm{CA}$ & Lepidopteran caterpillars \\
\hline Bacillus thuringiensis ssp. kurstaki 3a,3b var SA-12 & $\mathrm{B}$ & AUS (1996) & Cotton bollworm \\
\hline Bacillus thuringiensis ssp. kurstaki BMP123 & B & USA & Lepidopteran caterpillars \\
\hline Bacillus thuringiensis ssp. kurstaki EG & B & $\mathrm{BR}$ & Lepidopteran caterpillars \\
\hline Bacillus thuringiensis ssp. kurstaki EG2348 & $\mathrm{B}$ & USA & Lepidopteran caterpillars \\
\hline Bacillus thuringiensis ssp. kurstaki EG2371 & B & USA & Lepidopteran caterpillars \\
\hline Bacillus thuringiensis ssp. kurstaki EG7826 & $\mathrm{B}$ & USA & Lepidopteran caterpillars \\
\hline
\end{tabular}


Table 3 continued

Microorganism $^{\mathrm{a}}$

Bacillus thuringiensis ssp. kurstaki EG7841

Bacillus thuringiensis kurstaki evb-113-19

Bacillus thuringiensis ssp. kurstaki encapsulated in killed Pseudomonas fluorescens

Bacillus thuringiensis ssp. kurstaki h-3a,3b hd1

Bacillus thuringiensis ssp. kurstaki h-3a,3b, hd 263

Bacillus thuringiensis ssp. kurstaki h-3a,3b, SA-11

Bacillus thuringiensis ssp. kurstaki HD-1

Bacillus thuringiensis ssp. kurstaki SA-11

Bacillus thuringiensis ssp. kurstaki SA-12

Bacillus thuringiensis ssp. san diego encapsulated in killed Pseudomonas fluorescens

Bacillus thuringiensis ssp. tenebrionis NB 176

Beauveria bassiana 147

Beauveria bassiana 447

Beauveria bassiana ANT-03

Beauveria bassiana ATCC 74040

Beauveria bassiana CG 716

Beauveria bassiana GHA

Beauveria bassiana $\mathrm{HF} 23$

Beauveria bassiana IBCB 66

Beauveria bassiana IMI389521

Beauveria bassiana $\mathrm{k} 4 \mathrm{~b} 1$

Beauveria bassiana k4b3

Beauveria bassiana NPP111B005

Beauveria bassiana PL63

Beauveria bassiana PPRI 5339

Beauveria brongniartii NBL 851

Burkholderia (Pseudomonas) cepacia M54

Burkholderia (Pseudomonas) cepacia J82

Candida oleophila isolate I-182

Candida oleophila $\mathrm{O}$

Chondrostereum purpureum PFC 2139

Chromobacterium subtsugae PRAA4-1T

Clavibacter michiganensis ssp. michiganensis bacteriophage

Colletotrichum gloeosporioides $\mathrm{f}$. sp. aeschynomene

Condylorrhiza vestigialis NPV

Coniothyrium minitans CON/M/91-08

Cydia pomonella $\mathrm{GV}$ (Mexican strain and various other strains)

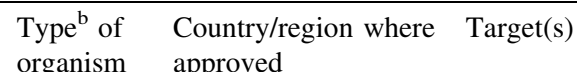

organism approved

$\begin{array}{lll}\text { B } & \text { USA } & \begin{array}{l}\text { Lepidopteran caterpillars } \\ \text { Lepidopteran caterpillars } \\ \text { B }\end{array} \\ \text { B } & \text { USA } & \text { Lepidopteran caterpillars } \\ \text { B } & \text { NZ (1996) } & \text { Lepidopteran caterpillars } \\ \text { B } & \text { NZ (2000) } & \text { Lepidopteran caterpillars } \\ \text { B } & \text { NZ (1995) } & \text { Lepidopteran caterpillars } \\ \text { B } & \text { AUS (2000), BR, CA } & \text { Lepidopteran caterpillars } \\ \text { B } & \text { AUS (2008) } & \text { Lepidopteran caterpillars } \\ \text { B } & \text { AUS (2005) } & \text { Cotton bollworm } \\ \text { B } & \text { USA } & \text { Beetles } \\ \text { B } & \text { CA, EU } & \text { Beetles } \\ \text { F } & \text { EU } & \text { Red palm weevil, soft bodied insects } \\ \text { F } & \text { USA } & \text { Ants }\end{array}$

F USA

$\mathrm{F} \quad \mathrm{CA}$

Ants

F EU, NZ (2013), USA

$\mathrm{F} \quad \mathrm{BR}$

Soft bodied insects

$\mathrm{BR}$

Spidermites, whitefly, thrips, aphids

Whitefly, spidermites, beetles

F CA, EU, J Whitefly, thrips, aphids

$\mathrm{F} \quad \mathrm{CA}$

F BR

$\mathrm{F} \quad \mathrm{EU}^{\mathrm{c}}$

F NZ (2005)

F NZ (2009)

$\mathrm{F} \quad \mathrm{EU}^{\mathrm{c}}$

$\mathrm{F} \quad \mathrm{Br}$

$\mathrm{F} \quad \mathrm{CA}, \mathrm{EU}^{\mathrm{c}}$

$\mathrm{F} \quad \mathrm{J}$

Y USA

Y USA

Y USA

Soft bodied insects

Whitefly, spidermites, beetles

Beetles in stored grain

Thrips

Sucking insects

Banana weevil, red palm weevil

Whitefly, spidermites, beetles

Soft bodies insects, caterpillars

Long horn beetle etc.

Damping off diseases, nematodes

Damping off diseases, nematodes

Post-harvest fungicide

Post-harvest fungicide

Inhibits sprouting/regrowth of shrubs and trees

Various insects and mites

Clavibacter michiganensis ssp. michiganensis

Northern jointvetch (Aeschynomene virginica)

Condylorrhiza vestigialis (Braz. poplar moth)

F CA, EU, USA Sclerotinia spp.

V AUS (2010), CA, EU, Codling moth NZ (1999), USA 
Table 3 continued

Microorganism $^{\mathrm{a}}$ $\begin{array}{lll}\begin{array}{l}\text { Type } \\ \text { organism of }\end{array} & \begin{array}{l}\text { Country/region where } \\ \text { approved }\end{array} & \text { Target(s) }\end{array}$

Cydia pomonella GV V22 (CPGV-V22)

Erwinia carotovora CGE234

Fusarium sp. L13

Gliocladium catenulatum J1446

Gliocladium virens $\mathrm{G}-21$

Helicoverpa armigera NPV

Helicoverpa zea NPV

Homona magnanima GV

Isaria fumosorosea Apopka 97 (formely Paecilomyces fumosoroseus)

Isaria fumosorosea $\mathrm{Fe} 9901$

Lactobacillus casei LPT-111

Lactobacillus plantarum BY

Lactobacillus rhamnosus LPT-21

Lactococcus lactis ssp. cremoris M11/CSL

Lactococcus lactis ssp. lactis LL102/CSL

Lagenidium giganteum

Lecanicillium lecanii (formerly Verticillium lecanii)

$\mathrm{K} 4 \mathrm{~V} 1+\mathrm{K} 4 \mathrm{~V} 2$

Lecanicillium lecanii (formerly Verticillium lecanii) K4V2

Lecanicillium muscarium (formerly Verticillium lecanii) Ve6

Lymantri dispar NPV

Metarhizium anisopliae

Metarhizium anisopliae

Metarhizium anisopliae var. acridum

Metarhizium anisopliae ESF1

Metarhizium anisopliae IBCB 348

Metarhizium anisopliae PL 43

Metarhizium anisopliae SMZ-2000

Metarhizium anisopliae var. anisopliae BIPESCO 5/F52

Metschnikowia fructicola NRRL Y-27328

Muscodor albus QST 20799

Myrothecium verrucaria dried fermentation solids and F solubles

Neodiprion abietis NPV

Neodiprion lecontei NPV

Nosema locustae

Orgyia pseudotsugata NPV

Paecilomyces lilacinus

Paecilomyces lilacinus 251

Paecilomyces tenuipes T1
V

V

F

F

B

B

B

B

B

F

F

F

F

V

F

F

F

F

F

F

F

F

F

Y

F

V

V

M

V

F

F

F

$\begin{array}{lll}\text { V } & \text { AUS (2015) } & \text { Codling moth } \\ \text { B } & \text { J } & \begin{array}{l}\text { Bacterial soft rot in potato and } \\ \text { vegetables }\end{array}\end{array}$

F $\quad \mathrm{EU}^{\mathrm{c}}$

F CA, EU, USA

No information found about target

USA

Foliar fungal diseases

Damping off diseases

AUS (2002), Br, EU, Helicoverpa ssp. USA

AUS (1999), Br, USA $\mathrm{J}$

EU, J, USA

CA, EU

$\mathrm{CA}$

$\mathrm{J}$

CA

$\mathrm{CA}$

CA

USA

NZ (2012)

NZ (2012)

EU, J

CA, USA

AUS

AUS

AUS

USA

$\mathrm{Br}$

$\mathrm{Br}$

J

CA, EU, USA

$\mathrm{EU}^{\mathrm{c}}$

USA

USA

CA

$\mathrm{CA}$

CA, USA

CA, USA

BR

EU

J
Helicoverpa ssp.

Tea leaf roller, tTea tortorix

Soft bodied insects

soft bodied insects

Various weeds in lawns

Soft rot

Various weeds in lawns

Various weeds in lawns

Various weeds in lawns

Mosquitoes

Thrips, whitefly, aphids, mealy bug, psyllid and passion vine hopper

Whitefly, thrips, aphids, passion vine hopper

Whitefly, thrips

Lymantra dispar

Redheaded pasture cockchafer

Greyback canegrub

Locusts

Termites

Leafhoppers

Leafhoppers

Aphids, thrips, whitefly

Black vine weevil, thrips

Post-harvest diseases

Bacteria, fungi, and nematodes

Nematodes

Balsam fir sawfly

Redheaded pine sawfly

Grasshoppers, locusts, crickets

Ddouglas-fir tussock moth

Root knot nematodes

Root knot nematodes

Whitefly, aphids, powdery mildew 
Table 3 continued

Microorganism $^{\mathrm{a}}$ $\begin{array}{lll}\begin{array}{l}\text { Type } \\ \text { organism }\end{array} & \begin{array}{l}\text { Country/region where } \\ \text { approved }\end{array} & \text { Target(s) }\end{array}$

Pantoea agglomerans $\mathrm{C} 9-1$

Pantoea agglomerans E325

Pantoea agglomerans $\mathrm{p} 10 \mathrm{c}$

Pasteuria nishizawae Pn1

Pepino mosaic virus $\mathrm{CH} 2$ isolate 1906

Pepino Mosaic Virus isolate VC 1

Pepino Mosaic Virus isolate VX 1

Phlebiopsis gigantea (several strains)

Phlebiopsis gigantea VRA 1992

Phoma macrostoma

Phytophthora palmivora MWV

Plodia interpunctella granulosis virus

Pochonia chlamydosporia PC10

Pseudomonas aureofaciens Tx-1

Pseudomonas chlororaphis 63-28

Pseudomonas chlororaphis MA342

Pseudomonas fluorescens G 7090

Pseudomonas fluorescens 1629RS

Pseudomonas fluorescens A506 (syn. 006418)

Pseudomonas fluorescens CL145A

Pseudomonas rhodesiae HAI-0804

Pseudomonas sp. DSMZ 13134

Pseudomonas syringae 742RS

Pseudomonas syringae ESC 10

Pseudomonas syringae ESC-11

Pseudozyma flocculosa PF-A22 UL

Puccinia thlaspeos

Purpureocilium lilacinum PL 11

Pythium oligandrum M1

Saccharomyces cerevisiae extract hydrolysate

Saccharomyces cerevisiae LAS02

Sclerotinia minor IMI 3144141

Serratia entomophila 626

Spodoptera exigua NPV

Spodoptera frugiperda NPV 3AP2

B

B

B

B

V

V

V

F

F

F

F

$\mathrm{V}$

F

B

B

B

B

B

B

B

B

B

B

B

B

F

F

F

F

Y

Y

F

B

V

V
CA, USA

$\mathrm{CA}$

NZ (2006)

$\mathrm{CA}, \mathrm{EU}^{\mathrm{c}}$, USA

EU

$\mathrm{EU}^{\mathrm{c}}$

$\mathrm{EU}^{\mathrm{c}}$

EU

CA

CA

USA

USA

BR

USA

USA

EU

J

USA

CA, USA

CA

$\mathrm{J}$

EU

USA

CA, USA

USA

$\mathrm{EU}^{\mathrm{c}}$, USA

USA

$\mathrm{EU}^{\mathrm{c}}$

$\mathrm{EU}$

USA

EU

CA

NZ (1994)

EU, USA

USA
Fire blight in apples and pears

Fire blight in apples and pears

Fire blight in apples and pears

Nematodes (Heterodera, Globodera)

Pepino mosaic virus

Pepino mosaic virus

pepino mosaic virus

Root run (Heterobasidion annosum) in conifers

Root run (Heterobasidion annosum) in conifers

Broadleaf weeds in turf grass

Strangler vine (Morenia orderata)

Plodia interpunctella

Nematodes

Fungal diseases in turf grass

Pythium spp., Rhizoctonia solani, Fusarium oxysporum

Seed-borne pathogens on barley and oats

Bacterial and black rot in lettuce/cabbage

Frost prevention in fruits, almond, potato, tomato

Frost prevention in fruits, almond, potato, tomato

Zebra mussel

Bacterial diseases in citrus, peach, plum

Rhizoctonia solani in potato

Frost prevention in fruits, almond, potato, tomato

Post-harvest diseases in various fruits

Post-harvest diseases in various fruits

Powdery mildew on roses and cucumbers

Isatis tinctoria, dyer's woad

Nematodes

Fungal diseases in cereals and oil seed rape

Bacterial diseases

Fungal diseases in fruits

Dandelion in turf

Grass grubs

Spodoptera exigua (beet army worm)

Spodoptera frugiperda 
Table 3 continued

\begin{tabular}{|c|c|c|c|}
\hline Microorganism $^{\mathrm{a}}$ & $\begin{array}{l}\text { Type }^{\mathrm{b}} \text { of } \\
\text { organism }\end{array}$ & $\begin{array}{l}\text { Country/region where } \\
\text { approved }\end{array}$ & Target(s) \\
\hline Spodoptera littoralis NPV & $\mathrm{V}$ & EU & $\begin{array}{l}\text { Spodoptera littoralis (cotton leaf } \\
\text { worm) }\end{array}$ \\
\hline Streptomyces acidiscabies RL-110T & $\mathrm{B}$ & $\mathrm{CA}$ & Dandelion on turf grass \\
\hline Streptomyces griseoviridis $\mathrm{K} 61$ & $\mathrm{~B}$ & CA, EU, USA & $\begin{array}{l}\text { Fungal soil diseases in vegetables, } \\
\text { ornamentals }\end{array}$ \\
\hline Streptomyces lydicus ATCC 554456 & $\mathrm{~B}$ & NZ (2013) & Soil borne and foliar diseases \\
\hline Streptomyces lydicus WYEC 108 & $\mathrm{~B}$ & $\begin{array}{l}\text { CA, EU, NZ (2009), } \\
\text { USA }\end{array}$ & Soil borne and foliar diseases \\
\hline Talaromyces flavus SAY-Y-94-01 & $\mathrm{F}$ & $\mathrm{J}$ & Fungal and bacterial diseases \\
\hline $\begin{array}{l}\text { Trichoderma asperellum (formerly } T \text {. harzianum) } \\
\text { ICC012 }\end{array}$ & $\mathrm{F}$ & EU & $\begin{array}{l}\text { Fungal soil diseases in vegetables, } \\
\text { ornamentals }\end{array}$ \\
\hline Trichoderma asperellum (formerly T.viride) T25 & $\mathrm{F}$ & EU & $\begin{array}{l}\text { Fungal soil diseases in vegetables, } \\
\text { ornamentals }\end{array}$ \\
\hline $\begin{array}{l}\text { Trichoderma asperellum (formerly T. harzianum) } \\
\text { TV1 }\end{array}$ & $\mathrm{F}$ & EU & $\begin{array}{l}\text { Fungal soil diseases in vegetables, } \\
\text { ornamentals }\end{array}$ \\
\hline Trichoderma asperellum SF 04 (URM) 5911 & $\mathrm{~F}$ & $\mathrm{BR}$ & Damping off, Sclerotinia sclerotiorum \\
\hline Trichoderma asperellum $\mathrm{T} 211$ & $\mathrm{~F}$ & $\mathrm{BR}$ & Damping off, Sclerotinia sclerotiorum \\
\hline Trichoderma asperellum T34 & $\mathrm{F}$ & $\mathrm{CA}, \mathrm{EU}$ & $\begin{array}{l}\text { Fungal soil diseases in vegetables, } \\
\text { ornamentals }\end{array}$ \\
\hline Trichoderma atroviridae SKT-1 & $\mathrm{F}$ & $\mathrm{J}$ & $\begin{array}{l}\text { Bacterial seedling blight and grain rot, } \\
\text { seedling fungal blight }\end{array}$ \\
\hline Trichoderma atroviride (5 strains) & $\mathrm{F}$ & NZ (1991) & Wound pathogens \\
\hline $\begin{array}{l}\text { Trichoderma atroviride (formerly T. harzianum) IMI } \\
206040\end{array}$ & $\mathrm{~F}$ & EU & $\begin{array}{l}\text { Fungal soil diseases in vegetables, } \\
\text { ornamentals }\end{array}$ \\
\hline Trichoderma atroviride (formerly T. harzianum) T11 & $\mathrm{F}$ & EU & $\begin{array}{l}\text { Fungal soil diseases in vegetables, } \\
\text { ornamentals }\end{array}$ \\
\hline Trichoderma atroviride ag1, ag2, ag3, ag5, ag11, ag15 & $\mathrm{F}$ & NZ (1987) & Wound pathogens \\
\hline Trichoderma atroviride I-1237 & $\mathrm{F}$ & EU & $\begin{array}{l}\text { Wound pathogens and fungal soil } \\
\text { diseases }\end{array}$ \\
\hline Trichoderma atroviride lu132 & $\mathrm{F}$ & NZ (2004) & Foliar diseases \\
\hline Trichoderma atroviride $\mathrm{SC} 1$ & $\mathrm{~F}$ & EU & Wound pathogens \\
\hline Trichoderma gamsii (formerly T. viride) ICC080 & $\mathrm{F}$ & EU & $\begin{array}{l}\text { Fungal soil diseases in vegetables, } \\
\text { ornamentals }\end{array}$ \\
\hline Trichoderma hamatum TH382 & $\mathrm{F}$ & USA & $\begin{array}{l}\text { Fungal soil diseases in vegetables, } \\
\text { ornamentals }\end{array}$ \\
\hline Trichoderma harzianum & $\mathrm{F}$ & AUS (2004) & Eutypa dieback in grapes \\
\hline Trichoderma harzianum KRL-AG2 (syn. T22) & $\mathrm{F}$ & CA, EU, USA & $\begin{array}{l}\text { Fungal soil diseases in vegetables, } \\
\text { ornamentals }\end{array}$ \\
\hline Trichoderma harzianum ESALQ-1306 & $\mathrm{F}$ & BR & Damping off, Sclerotinia sclerotiorum \\
\hline Trichoderma harzianum IBL F006 & $\mathrm{F}$ & BR & Damping off, Sclerotinia sclerotiorum \\
\hline Trichoderma harzianum ITEM 908 & $\mathrm{~F}$ & $\mathrm{EU}$ & Soil borne diseases \\
\hline Trichoderma harzianum T-39 & $\mathrm{F}$ & USA & $\begin{array}{l}\text { Fungal soil diseases in vegetables, } \\
\text { ornamentals }\end{array}$ \\
\hline Trichoderma polysporum ATCC 20475 & $\mathrm{~F}$ & USA & Wound pathogens \\
\hline Trichoderma polysporum IMI 206039 & $\mathrm{~F}$ & EU & $\begin{array}{l}\text { Botrytis cinerea, Chondrostereum } \\
\text { purpureum }\end{array}$ \\
\hline Trichoderma stromaticum CEPLAC 3550 & $\mathrm{~F}$ & BR & Witch's broom \\
\hline
\end{tabular}


Table 3 continued

\begin{tabular}{|c|c|c|c|}
\hline Microorganism $^{\mathrm{a}}$ & $\begin{array}{l}\text { Type }^{\mathrm{b}} \text { of } \\
\text { organism }\end{array}$ & $\begin{array}{l}\text { Country/region where } \\
\text { approved }\end{array}$ & Target(s) \\
\hline Trichoderma virens $\mathrm{G}-41$ & $\mathrm{~F}$ & $\mathrm{CA}$ & $\begin{array}{l}\text { Fungal soil diseases in vegetables, } \\
\text { ornamentals }\end{array}$ \\
\hline Trichoderma viride ATCC 20476 & $\mathrm{~F}$ & USA & Wound pathogens \\
\hline Typhula phacorrhiza 94671 & $\mathrm{~F}$ & $\mathrm{CA}$ & Snow molds in turf \\
\hline Ulocladium oudemansii U3 & $\mathrm{F}$ & NZ (2004) & $\begin{array}{l}\text { Foliar diseases, Pseudomonas } \\
\text { syringae }\end{array}$ \\
\hline $\begin{array}{l}\text { Verticillium albo-atrum (formerly V. dahliae) } \\
\text { WCS } 850\end{array}$ & $\mathrm{~F}$ & CA, EU, USA & Dutch elm disease \\
\hline $\begin{array}{l}\text { Xanthomonas campestris pv. vesicatoria } \\
\text { bacteriophage }\end{array}$ & $\mathrm{BP}$ & USA & $\begin{array}{l}\text { Xanthomonas campestris pv. } \\
\text { vesicatoria }\end{array}$ \\
\hline
\end{tabular}

Information obtained from (AUS) https://portal.apvma.gov.au/pubcris, (BR) http://extranet.agricultura.gov.br/agrofit_cons/principal_ agrofit_cons, (CA) http://pr-rp.hc-sc.gc.ca/ls-re/result-eng.php?p_search_label, (EU) http://ec.europa.eu/food/plant/pesticides/eupesticides-database/public/?event=homepage\&language=EN, (J) Japan Plant Protection Association, (NZ) https://eatsafe.nzfsa. govt.nz/web/public/acvm-register, (USA) https://iaspub.epa.gov/apex/pesticides/f?p=chemicalsearch:1

${ }^{\text {a }}$ Strain numbers if available

b $B$ bacterium, $B P$ bacteriophage, $F$ fungus, $Y$ yeast, $V$ virus

c Pending in the EU

Integrated Pest Management (IPM) program that provides an environmentally and economically sound alternative to chemical pest control (van Lenteren and Bueno 2003; Cock et al. 2010). Increasingly, seed treatments with microbial biological control agents are also used as a form of ABC (Abuamsha et al. 2011). We estimate that in $2015 \mathrm{ABC}$ was applied on more than 30 million ha worldwide (Table 1).

Since the 1970s, ABC has moved from a cottage industry to professional research and production facilities, as a result of which many efficient agents have been identified, quality control protocols, mass production, shipment and release methods matured, and adequate guidance for farmers has been developed (van Lenteren 2003, 2012; Cock et al. 2010; Ravensberg 2011). In this paper we will not describe the process of collection, evaluation, development of mass production and registration of biological control agents in detail. Information concerning these factors for invertebrate biological control agents can be found in Cock et al. (2010) and for microbial biological control agents in Köhl et al. (2011), Ravensberg (2011) and Parnell et al. (2016). When searching for natural enemies, it is not unusual to find dozens or more species attacking a certain pest, but criteria such as population growth rate, host range, and adaptation to crop and climate can often be used to quickly eliminate clearly inefficient species. The most promising species are compared by using characteristics such as efficacy of pest control, potential environmental risks and economy of mass rearing. For the screening of microbial control agents, large collections of hundreds or thousands of isolates are typically established and high throughput screening assays are increasingly applied to assess important traits such as cold tolerance, metabolite production and efficacy against the target pest.

Important recent successes in the use of $\mathrm{ABC}$ include the virtually complete replacement of chemical pesticides by predators (mites and hemipterans) to control thrips and whiteflies on sweet peppers in greenhouses in Spain (Calvo et al. 2012), and hemipteran predators to control new invasive pests like the South American pinworm Tuta absoluta (Meyrick) (Lepidoptera: Gelechiidae) (Urbaneja et al. 2012). These examples show how well biological control with invertebrate biological control agents can function in modern agriculture, and that they can actually save agriculture in large areas that otherwise would have had to terminate vegetable production. Another recent success deals with the importance of microbial control agents. The invasion of the cotton bollworm, Helicoverpa armigera (Hübner) (Lepidoptera: Noctuidae), into Brazil in 2012 caused tremendous damage to corn, cotton, and soy, as pesticides were not effective due to resistance, or 
were simply not available. Emergency approvals of the entomopathogenic bacterium Bacillus thuringiensis and baculovirus products provided farmers with the only effective control method at the time (Pratissoli et al. 2015).

Europe is still the largest commercial market for ABC with invertebrate biological control agents, which is partly due to political support of biological control within IPM programs (EC 2009), but also due to consumer demand, pressure by NGOs (e.g., Greenpeace 2007) and a well-functioning, highly developed biological control industry. The next largest market is North America, followed by Asia, Latin America, Africa and the Middle East. A strong growth of ABC with arthropods is taking place in Latin America and the same is expected to occur in Asia (Dunham 2015; ResearchandMarkets 2016b). According to the latest marketing reports (e.g., ResearchandMarkets 2016a) North America is now the largest market for biopesticides, followed by Europe.

\section{Commercially available biological control agents}

Cock et al. (2010) mentioned 170 species of invertebrate biological control agents that have been used in ABC in Europe. Van Lenteren (2012) provided a list of about 230 species of invertebrate biological control agents that have been used in pest management worldwide, but recognised that this list was not yet complete. Collection of new data in 2016 showed the use of almost 350 natural enemy species (Table in Supplementary electronic information). There are about 500 commercial producers of invertebrate biological control agents worldwide, although most of these employ less than ten people each. Less than ten producers employ more than 50 staff, with the largest producer having about 1400 employees. In addition to commercial producers, there are hundreds of government-owned production units, particularly in China, India and Latin America. Also, and especially in Latin America, some large-scale farmers and growers are involved in producing their own natural enemies. In addition to the species listed in Table 2, invertebrates are commercially produced for biological control of weeds (40 species), for soil improvement (six species), as feed and food (40 species), and as pollinators (ten species). These species are not listed in Table 2.
After predators and parasitoids, microbial biological control agents are the next most commonly used organisms in ABC. As far as we are aware, Table 3 may be the first list published about microbial biological control agents registered worldwide. Although we realize the list is not yet complete, it provides information on about 209 microbial strains from 94 different species commercially available for control of pests. Information we could obtain on registered strains was not always consistent. In some cases agents seem registered without strain information or under different strain identifications for different regions, so that some organisms may be listed more than once in Table 3. Microbial biological control agents are produced by approximately 200 manufacturers, but this is an underestimate as no data are available for China or India (Dunham 2015). There is a great diversity of manufacturers and often they are specialised in one or two types of microorganisms and production methods. The majority of manufacturers are small to medium-sized companies. Recently, large multinational agro-chemical companies are getting involved in the production and marketing of so-called biopesticides, again through the acquisition of small to medium-sized companies. New companies are still founded on a regular basis, and acquisitions and mergers occur frequently. There is a similar trend to consolidation as that which occurred in the seed and chemical pesticides business in the past decades.

\section{Viability of commercial biological control market}

Producers of natural enemies are understandably reluctant to provide data about market developments, profit margins and sales volumes. In 2015, the global pesticide market had a value of US\$ 58.46 billion (ResearchandMarkets 2016a). The global market of biological control agents (invertebrates and microorganisms) was approximately US\$ 1.7 billion in 2015 (Dunham 2015; Dunham W, personal communication 2016), which is less than $2 \%$ of the pesticide market. Growth of the market for synthetic pesticides is expected to be between 5 and $6 \%$ over the next five years (Research and Markets 2016b), but interestingly, growth of the biological control market has been faster: it showed an annual increase of sales of $10 \%$ before 2005 and more than $15 \%$ per year since 2005 (Dunham 2015; Dunham W, personal communication 
2016). The largest European biological control companies are still getting the main part of their income from sales of invertebrate biological control agents, but the contribution of microbial biological control agents is steadily increasing. Commercial $\mathrm{ABC}$ is used in protected crops (e.g., vegetables, ornamentals) and high-value outdoor crops (e.g., strawberries, vineyards), contributing to about $80 \%$ of the market value of invertebrate biological control agents. Biological control programs for each of these crops may involve up to 15-20 different species of natural enemies (van Lenteren 2000). The remaining $20 \%$ of the market value for natural enemies comes from application of relatively simple, cheap but effective biological control programs often using only one biological control agent (e.g., Trichogramma spp. against lepidopterans in cereals and sugarcane, and Cotesia spp. against lepidopterans in sugarcane). Almost $40 \%$ of the income of the European companies originates from invertebrate biological control agents sold for control of thrips, another $30 \%$ for control of whitefly, $12 \%$ for control of spider mites, $8 \%$ for control of aphids, and the remaining $10 \%$ for control of various other pests (Bolckmans K, personal communication 2016). Since 2005 , predatory mites have contributed enormously to the growth of the market for invertebrate biological control agents as a result of: the (re)discovery of their use for control of whiteflies (Nomikou et al. 2001), finding more efficient species for thrips control (Messelink et al. 2006), the development of techniques to enhance dispersal and establishment of predatory mites in crops (Messelink et al. 2014), and the development of new highly economic production technologies (Bolckmans et al. 2005).

The recent increase in annual market growth for biological control agents is the result of many factors. Compared with synthetic chemical pesticides, ABC agents show important inherent positive characteristics: they are less detrimental to the health of farm workers and persons living in farming communities; they do not have a harvesting interval or re-entry period as do pesticides; they are more sustainable, as there has been no development of resistance against arthropod ABC agents; they do not cause phytotoxic damage to plants and, as a result, most farmers report better yields and healthier crops after switching to biocontrol-based IPM. Increasingly, produce in Europe and North America can only be sold when residue levels are well below the legal MRLs because of retailers demands. In some cases low residue levels give farmers a preferred partnership with retailers who prefer to buy products with less residues. Furthermore, biological control might contribute to considerable reduction in emission of greenhouse gasses in comparison with pesticide use (Heimpel et al. 2013).

In addition to these inherent advantages of biological control, consumers have and will increasingly express concerns about food safety and environmental impact issues in relation to synthetic pesticide use, though they often have no direct way to influence crop protection policies. However, food retailers and supermarkets cleverly exploit this and use these two concerns increasingly in advertising their produce. In many countries, retailers and supermarkets more strongly restrict use of pesticides than do government policies (Buurma et al. 2012), and, particularly in Europe, the effect of NGOs reporting on excess residue levels and illegal use of pesticides has had a positive effect on the use of biological control (e.g., Greenpeace 2007). Adoption of IPM programs in the $\mathrm{EU}$, in which biological control is a cornerstone, has increased interest in and application of ABC (Lamichhane et al. 2017). Concurrently with the adoption of this IPM approach, it was announced that a large number of pesticides were to be legally discontinued and this has also led to requests for $\mathrm{ABC}$ solutions. Policy measures such as a strong reduction in use of synthetic pesticides in China have also opened avenues for $\mathrm{ABC}$. A number of other national measures have been shown to stimulate use of ABCs. Examples are fast track and priority registration of low risk pest control agents such as ABC's similar to the special registration procedure for biopesticides in the USA (EPA 2017), subsidizing biological control agents to growers (several countries in the EU) and application of pesticide levies (e.g., Denmark).

However, ABC, and biological control in general, also face a very serious problem. Pests have been accidentally exported for many years, but at an ever increasing rate (Bacon et al. 2012). Until recently, potential biological control agents could be collected in the country of origin of the pest, evaluated, mass produced and released when an effective agent was found. But today, under the Convention on Biological Diversity (CBD 1993) countries have sovereign rights over their genetic resources and agreements governing the access to these resources and the sharing of 
benefits arising from their use need to be established between involved parties [i.e., Access and Benefit Sharing (ABS) (Cock et al. 2010)]. This means that currently, permission to sample potential biological control agents can only be granted by the country that has sovereign rights over the genetic resources and collection of new natural enemies has become increasingly difficult or impossible in countries which have first accidentally exported the pest, a situation which seems very unreasonable.

\section{What might boost future use of $\mathrm{ABC}$ ?}

First, we expect that the above-mentioned factors that are responsible for the recent growth of $\mathrm{ABC}$ will play an even more important role in the near future, as their influence will spread to other countries and regions worldwide due to support for "greener" agriculture by consumers, NGOs, governments and growers. Another influential issue accelerating use of $\mathrm{ABC}$ relates to changing regulations. Regulations should facilitate the use of innovative sustainable solutions resulting in a choice for the ecological best pest control option. This can be realized by fast track registration, priority registration, and use of a combination of comparative assessment of pest control methods together with the substitution principle, through which an environmentally safer pest control method can substitute for a synthetic pesticide (EC 2009). Also zonal authorization (e.g., authorization for all of the EU instead of registration per country), permanent registration (instead of reregistration after 10-15 years) and mutual recognition of registration by member states in the EU are all measures that are likely to result in increased application of microbial biological control, and are now considered for low risk substances including ABC's in the EU. The changes in registration procedures will result in faster registration of more microbial biological control agents and, logically, in lower product costs. The Environmental Protection Agency (EPA) in the USA is already applying several of the above-mentioned factors related to registration of $\mathrm{ABC}$ 's, but the EU is slow in adopting specific criteria and procedures for biological control agents. Development of a specific protocol for registration of microbial biological control agents that will be used locally or worldwide, would be another big step forward in making use of biological control more attractive and accessible for farmers.

Removal of pesticides from the market due to observed health, non-target and environmental effects (e.g., the recent development concerning neonicotinoids; EASAC 2015), the development of resistance that makes pesticides less effective, and the appearance of new pests for which no pesticides are available (e.g., Tuta absoluta invasion in Europe in 2006, Urbaneja et al. 2012) all stimulate use of ABC. Nongovernmental organizations (NGOs) have in several cases successfully used information about environmental effects and illegal use of pesticides to initiate a change from chemical to biological control [e.g., in 2005 in the Almeria region in Spain, chemical control of pests in sweet pepper was replaced by biological control in a period of two years (Calvo et al. 2012)]. A very fast change from chemical control to biological control as in sweet peppers in Spain also occurred for other crops in that region. In the 1980s there was a similar drastic change from chemical to biological control in vegetable production in Northwest Europe (van Lenteren 2000), though this was not caused by a pesticide scandal like the one concerning sweet pepper production in Spain (Greenpeace 2007), but by growers recognition of the inherent positive characteristics of biological control mentioned above, and by resistance of several insect pests to conventional chemical pesticides. The development of new and better biological control solutions, improved and more stable formulations for microbial biological control agents and their use as seed treatments, more convenient application methods for invertebrate biological control agents (equipment to release biological control agents in crops, use of drones, etc.) and increasingly stable formulations of microbial biological control agents, have also contributed to growth in uptake of biological control. Interestingly, growers quickly took up the extra knowledge and methods to make biological control a success, and in quite a number of cases came up with new insights and technologies to improve release and establishment of invertebrate biological control agents. They also stimulated researchers and the biological control industry to provide new invertebrate biological control agents for emerging pests. We hope farmer's organizations will create a new renaissance in crop protection by seeing the many positive sides, including economics, of ABC. In their own interest they should become much 
more proactive and demand priority and fast track registration of innovative sustainable control methods.

Finally, application of the "true cost" principle for chemical pesticides would strongly increase the market for biological control. Pesticides are subsidized by governments because the industry is not held responsible for human illnesses and deaths as a result of chronic exposure to pesticides, and also does not have to provide the funding to repair damage done to the environment (e.g., reduction of biodiversity, limiting or even preventing the functioning of ecosystem services such as pest and disease control, pollination and cleaning of (drinking) water). Thus, pesticide costs related to harmful effects on human health and the environment are externalized and are actually paid by society, which is unethical and unscrupulous because the pesticide industry only reaps the economic benefits without being responsible for these costs. Benefit-cost ratios of chemical pesticides are usually said to be in the order of 4 when these "external hidden" costs are not taken into account (e.g., Pimentel and Burgess 2014). If true costs were applied to pesticides their benefit-cost ratio would still in most cases be higher than 1 , in other cases close to 1 , and in some cases even below 1, and, according to Bourguet and Guillemaud (2016) "the profitability of pesticides has, indeed, been overestimated in the past." Realistic pricing involving true costs would result in much higher costs of chemical pesticides and fairer competition with non-chemical alternative controls. Although hidden costs of pesticides have been documented since the 1980s, they have seldom resulted in an increase of pesticide prices. A first step to true cost pricing would be to apply levies on synthetic pesticides resulting both in higher, thus more realistic pricing, as well as in fairer competition with prices of biological control agents used in IPM programs.

\section{And what next?}

Too often the following reasoning is used to justify the use of synthetic pesticides: agriculture has to feed some ten billion people by the year 2050 , so we need to strongly increase food production, which can only be achieved with usage of synthetic pesticides. This reasoning is simplistic, erroneous and misleading. Simplistic because it ignores a multitude of other approaches to pest, disease and weed control that we summarize below under IPM, erroneous as sufficient healthy food can be produced without synthetic pesticides (e.g., IPES-Food 2016; Ponisio et al. 2014; UN 2017), and misleading in that it minimizes the importance of a well-functioning biosphere and high biodiversity for the long-term sustainable production of healthy food for a growing human population (De Vivo et al. 2016; Erisman et al. 2016; IPESFood 2016; Tillman et al. 2012). This short-sighted mercenary attitude might actually result in very serious environmental problems in the near future (e.g., van Bavel 2016). A more sensible approach to food production is to ask ourselves: (1) how can we create a healthy and well-functioning biosphere in which biodiversity is treasured instead of strongly reduced, both because of its necessity for sustainable food production and maintaining a hospitable biosphere for humans (utilitarian approach), as well as because of our ethical responsibility (ethical approach), (2) how can healthy food best be produced in this well-functioning biosphere, and (3) what kind of pest, disease and weed management fits in such a production system.

From the time agriculture developed some 10,000 years ago until only 65 years ago, agriculture was, after periods with slash and burn activities, an holistic activity, based on a systems approach. Farming societies had to design plant production and crop protection programs based on prevention of pests. This true form of IPM included, among others, planning of crop combinations, crop rotation, tillage, use of resistant or tolerant crop cultivars, choice of the right planting and harvesting periods, biological, mechanical and physical control etc. (e.g., Ehler 2006). Due to an understanding of plant genetics, the development of synthetic fertilizers and pesticides, agricultural research changed from an holistic approach to an extremely reductionist science where pests are avoided by a prophylactic approach consisting of calendar sprays or by curative treatments. A total systems approach to agriculture no longer seemed necessary, but this is a short-sighted and dangerous viewpoint and the ever increasing use of synthetic pesticides has resulted in a serious loss of biodiversity (e.g., EASAC 2015), which in turn resulted in prevention or reduction in functioning of the ecosystem services of pest reduction, pollination and water purification (Millennium Ecosystem Assessment 2005). A prophylactic approach is also an exorbitant 
input of resources with financial consequences of billions of US\$ (Costanza et al. 1997; Pimentel and Burgess 2014; Bourguet and Guillemaud 2016).

Lewis et al. (1997) made a plea to return to a system approach based on true IPM. True IPM is a durable, environmentally and economically justifiable system in which pest damage is prevented through the use of natural factors limiting pest population growth, and only-if needed-supplemented with other, preferably non-chemical measures (Gruys, P. in van Lenteren 1993). As stated above, there are many alternatives for synthetic pesticides, and cultural methods together with modern plant breeding and biological control within true IPM programs have been shown to provide excellent yields (e.g., Radcliffe et al. 2009). The fact that more creativity, knowledge and ecological insight are needed to be able to apply such pesticide-free crop management schemes should no longer be an excuse to use unsustainable, environmentally unsafe and toxic synthetic pesticide programs (UN 2017). We are not advocating a dogmatic, one-sided pest control approach, and we also do not support a static holistic approach in which (agro-) ecosystems are seen as non-changing functional units. Instead we propose to combine the sustainability gain from all types of agriculture and pest prevention/control methods, and consider agro-ecosystems as constantly changing systems. In such an approach, we are convinced that $\mathrm{ABC}$ can be applied much more than it is today, but we also know it will not solve all pest problems. A seriously neglected form of biological control, conservation biological control, should be the basis of most crop protection programs by providing sufficient invertebrate biological control agents and undisturbed buffering microbiomes in soils and plants when pests invade an agro-ecosystem (Gruys 1982; Blommers 1994; Berendsen et al. 2012). Delaying or preventing sprays will result in the reduction of secondary pests that arise after killing natural enemies of pest organisms. These often cause resurgence problems when synthetic pesticides are used. Hostplant resistance is one of the important cornerstones of IPM and should play a more important role in pest prevention. In IPM we are not dependent on full resistance, often partial host-plant resistance is enough because pest populations develop more slowly and natural enemies can more easily reduce such populations. Both classic and modern plant breeding, including CRISPR-Cas and RNAi, will help us design robust IPM programs. In order to obtain more governmental and public support, we-researchers and practitioners of biological control-will have to collaborate with all stakeholders in pest management to involve them and make them aware of the important economic, environmental, societal and environmental benefits of biological control. Recent experiences in New Zealand, where farmers pushed for and implemented biological control (Hardwick et al. 2016), protected crops in South-eastern Spain (growers and public embraced biological control: Jacas and Urbaneja 2009) and weed control in South Africa (public supported weed biological control in the working for water program: Moran et al. 2005) shows that widely disseminated information about successes of biological control projects result in strong public support and increased government funding.

In conclusion, we see the urgent need for a new type of agriculture that is somewhere between conventional and organic, is flexible and non-dogmatic. We might address it as Conscious Agriculture, a term which we borrowed from the conscious capitalism movement (Mackey and Sissodia 2014). Conscious agriculture involves participation of all stakeholders in the production and consumption chain, and respects the environment and resource availability for future generations. This is in contrast with conventional agriculture which concentrates on profit maximization and externalizing the cost of the harmful effects on human health, society and the environment (Robinson 2007; Erisman et al. 2016). Conscious agriculture fits seamlessly into a "common agricultural and food policy" as recently published in a position paper by Fresco and Poppe (2016). They review societal challenges and options for innovation, and conclude that such a policy should not concentrate on agriculture only, but needs to be developed with participation of all stakeholders, and will help "the entire food chain-from farm to fork, from animal breeding to human food production-to cope with the challenges of the coming decades". Within conscious agriculture, the first line of crop protection consists of strictly enforced quarantine regulations, prevention of pest development by cultural methods, host-plant resistance, classical and conservation biological control, preventative releases of natural enemies (an aspect of $\mathrm{ABC}$ ) and use of banker plants to establish natural enemy populations before pests establish (Messelink et al. 2014). When pests exceed acceptable population 
levels, i.e. when economic damage is expected to occur, augmentative biological control should be the first option for pest management, if needed in combination with other IPM tactics. Were "conscious agriculture" to be considered a serious alternative to conventional farming, augmentative biological control would face an even brighter future.

Acknowledgements The following persons/organizations are thanked for corrections and additions to Tables 2 and 3: ABC Bio (Brazil), Wagner Bettiol (Embrapa, Brazil) Vanda H.P. Bueno (ESALQ/USP, Brazil), Patrick DeClercq (Ghent University, Belgium), William Dunham (DunhamTrimmer, USA), Philip Elmer (Plant \& Food Research, Hamilton, New Zealand), Tom Groot (Koppert Biosystems, The Netherlands), Tobias Laengle (Agriculture and Agri-Food Canada), Lynn LeBeck (ANBP, USA), Li Liying (Guandong Entomological Institute, China), Antoon Loomans (NVWA,The Netherlands), Ricardo Machado and Danilo Pedrazzoli (Koppert Biosystems, Brazil), Dean Metcalf (Metcalf Bio Control, Australia), José R.P. Parra (ESALQ/USP), Amy Roberts (TSG, USA), Tetsuo Wada (Arysta, Japan). The participants of the IOBC Global Workshop on "Biological Control: Concepts and Opportunities" (October 2015, Engelberg, Switzerland) are thanked for the suggestions related to factors stimulating use of Augmentative Biological Control, as are Barbara Barratt, Russell Messing and two anonymous reviewers for improving the manuscript.

Open Access This article is distributed under the terms of the Creative Commons Attribution 4.0 International License (http:// creativecommons.org/licenses/by/4.0/), which permits unrestricted use, distribution, and reproduction in any medium, provided you give appropriate credit to the original author(s) and the source, provide a link to the Creative Commons license, and indicate if changes were made.

\section{References}

Abuamsha R, Salman M, Ehlers R-U (2011) Effect of seed priming with Serratia plymuthica and Pseudomonas chlororaphis to control Leptosphaeria maculans in different oilseed rape cultivars. Eur J Plant Pathol 130:287-295

Aristizabal LF, Bustillo AE, Arthurs SP (2016) Integrated pest management of coffee berry borer: strategies from Latin America that could be useful for coffee farmers in Hawaii. Insects 7(1):6. doi:10.3390/insects7010006

Bacon SJ, Bacher S, Aebi A (2012) Gaps in border controls are related to quarantine alien insect invasions in Europe. PLoS ONE 7(10):e47689

Berendsen RL, Pieterse CM, Bakker PA (2012) The rhizosphere microbiome and plant health. Trends Plant Sci 17:478-486

Blommers LHM (1994) Integrated pest management in European apple orchards. Annu Rev Entomol 39:213-241

Bolckmans KJF, Houten YM, van Baal AE, Castagnoli M, Nannelli R, Simoni S (2005) Mite composition comprising
Glycyphagidae and phytoseiid mites, use thereof, method for rearing a phytoseiid predatory mite, rearing system for rearing said phytoseiid predatory mite and methods for biological pest control on a crop. Patent registered as PCT/ NL2005/000899, Priority date: December 29, 2005

Bourguet D, Guillemaud T (2016) The hidden and external costs of pesticide use. In: Lightfouse E (ed) Sustanable agriculture reviews. Springer, Dordrecht, pp 35-120

Buurma JS, Lamine C, Haynes I (2012) Transition to consumerdriven value chains in The Netherlands. Acta Hortic 2012:69-76

Calvo FJ, Bolckmans K, Belda JE (2012) Biological controlbased IPM in sweet pepper greenhouses using Amblyseius swirskii (Acari: Phytoseiidae). Biocontrol Sci Technol 22:1398-1416

CBD (1993) Convention on biological diversity (with annexes). Concluded at Rio de Janeiro on 5 June 1992. United Nations-Treaty Series 1760 (30619):142-382

Cock MJW, van Lenteren JC, Brodeur J, Barratt BIP, Bigler F, Bolckmans K, Cônsoli FL, Haas F, Mason PG, Parra JRP (2010) Do new access and benefit sharing procedures under the convention on biological diversity threaten the future of biological control? BioControl 55:199-218

Costanza R, d'Arge R, de Groot R, Farber S, Grasso M, Hannon B, Limburg K, Naeem S, O’Neill RV, Paruelo J, Raskin RG, Sutton P, van den Belt M (1997) The value of the world's ecosystem services and natural capital. Nature 387:253-260

De Vivo R, Marchis A, Gonzalez-Sanchez EJ, Capri E (2016) The sustainable intensification of agriculture. Solutions 7:24-31

DeBach P (1964) Biological control of insect pests and weeds. Chapman and Hall, London

Dunham WC (2015) Evolution and future of biocontrol. Paper presented at the 10th Annual Biocontrol Industry Meeting (ABIM), Basel, Switzerland, October 20th, 2015. http:// www.abim.ch/index.php?eID=tx_nawsecuredl $\& \mathrm{u}=0 \& \mathrm{~g}=$ $0 \& \mathrm{t}=1489234639 \&$ hash $=9 \mathrm{a} 70 \mathrm{~d} 39 \mathrm{f} 93 \mathrm{f} 7 \mathrm{e} 559 \mathrm{c} 74 \mathrm{c} 63844 \mathrm{ae}$ 047a9aa3c37ea\&file=fileadmin/abim/documents/present ations2015/Keynote_Dunham_ABIM_2015.pdf. Accessed 10 Mar 2017

EASAC (2015) Ecosystem services, agriculture and neonicotinoids. EASAC policy report, 26 April 2015

EC (2009) Sustainable Use Directive. European Parliament and of the Council of 21 October 2009 establishing a framework for community action to achieve the sustainable use of pesticides. Off J Eur Union L309:71-86

Ehler LE (2006) Integrated pest management (IPM): definition, historical development and implementation, and the other IPM. Pest Manag Sci 62:787-789

Eilenberg J, Hajek A, Lomer C (2001) Suggestions for unifying the terminology in biological control. BioControl 46:387-400

EPA (2017) https://www.epa.gov/pesticides/biopesticides\# registration. Accessed $14 \mathrm{Feb} 2017$

Erisman JW, van Eekeren N, de Wit J, Koopmans C, Cuijpers W, Oerlemans N, Koks BJ (2016) Agriculture and biodiversity: a better balance benefits both. AIMS Agric Food 1:157-174

FAO/IPPC (1997) International plant protection convention. FAO, Rome 
Fresco L, Poppe K (2016) Towards a common agricultural and food policy. Wageningen University and Research, Wageningen. doi:10.18174/390280. Accessed 11 Nov 2016

Greenpeace (2007) Essen ohne Pestizide, Einkaufsratgeber für Obst und Gemüse. Greenpeace e. V, Hamburg

Gruys P (1982) Hits and misses. The ecological approach to pest control in orchards. Entomol Exp Appl 31:70-87

Gurr GM, Wratten SD (2000) Measures of success in biological control. Kluwer Academic Publishers, Dordrecht

Hardwick S, Ferguson CM, McCauley P, Nichol W, Barton DM, McNeill MR, Philip BA, Phillips CB (2016) Response to clover root weevil outbreaks in South Canterbury, Otago and Southland; the agricultural sector and government working together. J N Z Grass1 78:117-122

Heimpel GE, Yang Y, Hill JD, Ragsdale DW (2013) Environmental consequences of invasive species: greenhouse gas emissions of insecticide use and the role of biological control in reducing emissions. PLoS ONE 8(8):e72293

IPES-Food (2016) From uniformity to diversity: a paradigm shift from industrial agriculture to diversified agroecological systems. International Panel of Experts on Sustainable Food systems. http://www.ipes-food.org/reports. Accessed 10 Mar 2017

Jacas JA, Urbaneja A (2009) Control biológico de plagas agrícolas. Phytoma España, Valencia

Köhl J, Postma J, Nicot P, Ruocco M, Blum B (2011) Stepwise screening of microorganisms for commercial use in biological control of plant pathogenic fungi and bacteria. Biol Control 57:1-12

Lamichhane JR, Bischoff-Schaefer M, Blumel S, DachbrodtSaaydeh S, Dreux L, Jansen JP, Kiss J, Köhl J, Kudsk P, Malausa T, Nicot P, Ricci P, Thibierge J, Villeneuve F (2017) Identifying obstacles and ranking common biological control research priorities for Europe to manage most economically important pests in arable, vegetable and perennial crops. Pest Manage Sci 73:14-21

Lewis WJ, van Lenteren JC, Phatak SC, Tumlinson JH (1997) A total systems approach to sustainable pest management. PNAS 94:12243-12248

Liu SS, Rao A, Vinson SB (2014) Biological control in China: past, present and future-An introduction to this special issue. Biol Control 68:1-5

Lorito M, Woo SL, Harman GE, Monte E (2010) Translational research on Trichoderma: from 'omics to the field. Annu Rev Phytopathol 48:395-417

MacBain Cameron JW (1973) Insect pathology. In: Smith RF, Mittler TE, Smith CN (eds) History of entomology. Annual Reviews Inc. Palo Alto, pp 223-306

Mackey J, Sissodia R (2014) Conscious capitalism. Harvard Business Review Press, Boston

Mendes R, Kruijt M, De Bruijn I, Dekkers E, van Der Voort M, Schneider JHM, Piceno YM, DeSantis TZ, Andersen GL, Bakker PAHM, Raaijmakers JM (2011) Deciphering the rhizosphere microbiome for disease-suppressive bacteria. Science 332:1097-1100

Messelink GJ, van Steenpaal SEF, Ramakers PMJ (2006) Evaluation of phytoseiid predators for control of western flower thrips on greenhouse cucumber. BioControl 51:753-768

Messelink GJ, Bennison J, Alomar O, Ingegno BL, Tavella L, Shipp L, Palevsky E, Wäckers FL (2014) Approaches to conserving natural enemy populations in greenhouse crops: current methods and future prospects. BioControl 59:377-393

Millennium Ecosystem Assessment (2005) Ecosystems and human well-being: synthesis. Island Press, Washington, DC

Moran VC, Hoffmann JH, Zimmermann HG (2005) Biological control of invasive alien plants in South Africa: necessity, circumspection and success. Front Ecol Environ 3:77-83

Nomikou N, Janssen A, Schraag R, Sabelis MW (2001) Phytoseiid predators as potential biological control agents for Bemisia tabaci. Exp Appl Acarol 25:271-291

Parnell JJ, Berka R, Young HA, Sturino JM, Kang Y, Barnhart DM, DiLeo MV (2016) From the lab to the farm: an industrial perspective of plant beneficial microorganisms. Front Plant Sci 7:1110. doi:10.3389/fpls.2016.01110

Pimentel D, Burgess M (2014) Environmental and economic costs of the application of pesticides primarily in the United States. In: Pimentel D, Peshin R (eds) Integrated pest management. Springer, Dordrecht, pp 47-71

Ponisio LC, M'Conigle LK, Mace KC, Palomino J, de Valpine P, Kremen C (2014) Diversification practices reduce organic to conventional yield gap. Proc R Soc B 282:2014396

Pratissoli D, Lima VLS, Pirovani VD, Lima WL (2015) Occurrence of Helicoverpa armigera (Lepidoptera: Noctuidae) on tomato in the Espírito Santo state. Hortic Bras 33:101-105

Radcliffe EB, Hutchinson WD, Cancelado RE (eds) (2009) Integrated pest management: concepts, tactics, strategies and case studies. Cambridge University Press, Cambridge, UK

Ravensberg WJ (2011) A roadmap to the successful development and commercialization of microbial pest control products for control of arthropods. Springer, Dordrecht

ResearchandMarkets (2016a) Global pesticides market segmented by type, application area and geography. Trends and forecasts (2015-2020). Sustainability, regulation \& competition. http://www.researchandmarkets.com/resea rch/4hd338/global_pesticides. Accessed 10 Mar 2017

ResearchandMarkets (2016b) Biopesticides global strategic business report. http://www.researchandmarkets.com/ publication/mlv3aqe/347972. Accessed 10 Mar 2017

Robinson RA (2007) Self-organising agro-ecosystems. Sharebooks, Canada

Tillman D, Reich P, Isbell F (2012) Biodiversity impacts ecosystem productivity as much as resources, disturbance or herbivory. PNAS 109:10394-10397

UN (2017) Report of the special rapporteur on the right to food. Thirty-fourth session of the Human Rights Council 27 February-24 March 2017. Report UN A/HRC/38. http://ap. ohchr.org/documents/dpage_e.aspx?m=101. Accessed 10 Mar 2017

Urbaneja A, Gonzalez-Cabrera J, Arno J, Gabarra R (2012) Prospects for the biological control of Tuta absoluta in tomatoes of the Mediterranean basin. Pest Man Sci 68:1215-1222

van Bavel B (2016) The invisible hand? How market economies have emerged and declined since AD 500. Oxford University Press, Oxford

van Lenteren JC (1993) Integrated pest management: the inescapable future. In: Zadoks JC (ed) Modern crop protection: 
developments and perspectives, Wageningen Pers, Wageningen, pp 217-225

van Lenteren JC (2000) A greenhouse without pesticides: fact of fantasy? Crop Prot 19:375-384

van Lenteren JC (ed) (2003) Quality control and production of biological control agents: theory and testing Procedures. CABI, Wallingford

van Lenteren JC (2012) The state of commercial augmentative biological control: plenty of natural enemies, but a frustrating lack of uptake. BioControl 57:1-20

van Lenteren JC, Bueno VHP (2003) Augmentative biological control of arthropods in Latin America. BioControl 48:123-139

van Lenteren JC, Godfray HCJ (2005) European science in the enlightenment and the discovery of the insect parasitoid life cycle in The Netherlands and Great Britain. Biol Control 32:12-24

Waage JK, Greathead DJ (1988) Biological control: challenges and opportunities. Phil Trans R Soc Lond B 318:111-128

Wang ZY, He KL, Zhang F, Lu X, Babendreier D (2014) Mass rearing and release of Trichogramma for biological control of insect pests of corn in China. Biol Control 68:136-144

Weller DM, Raaijmakers JM, McSpadden Gardener BB, Thomashow LS (2002) Microbial populations responsible for specific soil suppressiveness to plant pathogens. Annu Rev Phytopathol 40:309-348

Yang NW, Zang LS, Wang S, Guao JY, Xu HX, Zhang F, Wan FH (2014) Biological pest management by predators and parasitoids in the greenhouse vegetables in China. Biol Control 68:92-102
Joop C. van Lenteren works on behavioural ecology and population dynamics of parasitoids and predators, theoretical and practical aspects of biological control, IPM and sustainable crop production.

Karel Bolckmans chief operating officer at BioBest Belgium, is passionate about biological control and sustainability and has long-term experience in applied biological control and IPM.

Jürgen Köhl is senior plant pathologist at Wageningen University \& Research, The Netherlands, working on the development of new microbial biological control agents for the control of fungal plant pathogens.

Willem J. Ravensberg works as biocontrol researcher (entomologist, insect-pathologist) at Koppert Biological Systems as Regulatory and Governmental Affairs Manager promoting biocontrol, IPM and sustainable agriculture.

Alberto Urbaneja is a senior researcher focused on biological control at Instituto Valenciano de Investigaciones Agrarias (IVIA, Spain), where his goal is to move citrus and vegetable pest management towards techniques based on biological control. 